\title{
Down-Regulation of MiR-1294 is Related to Dismal Prognosis of Patients with Esophageal Squamous Cell Carcinoma through Elevating C-MYC Expression
}

\author{
Kai Liu ${ }^{a}$ Liyi Lib Aizemaiti Rusidanmu Yongqing Wang ${ }^{c}$ Xiayi Lvc \\ aDepartment of Thoracic Surgery Ningbo, Medical Treatment Centre Lihuili Hospital, Ningbo, \\ bDepartment of General Surgery, The Second Affiliated Hospital \& Yuying Children's Hospital of \\ Wenzhou Medical University, Wenzhou, 'Department of Thoracic Surgery, First Affiliated Hospital of \\ Zhejiang University, Hangzhou, China
}

\section{Key Words}

MicroRNA • Esophageal squamous cell carcinoma $\bullet$ C-MYC $・$ Prognosis

\begin{abstract}
Aims: Changes in the expression of microRNAs (miRNAs) have been found in many cancers. This study aimed to investigate the expression of miR-1294 in patients with esophageal squamous cell carcinoma (ESCC) and its effect on prognosis. The underlying mechanism was explored as well. Methods: We examined the expression of miRNA in human ESCC cancer tissues and adjacent non-tumor controls using quantitative reverse transcription polymerase chain reaction (qRT-PCR). And the relationship between expressions of miR-1294 and ESCC prognosis was analyzed in this study. Over-expression and knock-down methods were used to investigate the biological functions of miRNA-1294. The effect of miRNA-1294 on cell proliferation was evaluated by MTT. Besides, the function of miR-1294 on cell migration and invasion were evaluated by transwell assays. Results: MiR-1294 was significantly downregulated in human ESCC tissues compared with the non-tumor controls tissues $(P=0.014)$. And patients with low miR-1294 expression had a significantly poorer prognosis than those with a high miR-1294 expression $(P=0.040)$. Negative association was defined between the expression of miR-1294 and the c-MYC expression in ESCC patients (Pearson correlation, $r=-$ $0.299, \mathrm{P}=0.0079$ ). Additionally, it was found that miR-1294 suppress esophageal cancer cells proliferation, migration and invasion capacity through targeting C-MYC in vitro. Conclusions: Down-regulation of miR-1294 correlates with poor prognosis of ESCC. It's partially due to the reduced function of c-MYC. This study may give insight into the understanding of pathogenesis of esophageal cancer and provide evidence for diagnosis and treatment of esophageal cancer.

K. Liu and L. Li contributed equally to this work.

Xiayi Lv

KARGER 125 


\section{Introduction}

Esophageal cancer is now the eighth most common cancer and the sixth most common cause of cancer deaths worldwide. Histologically, in approximately $95 \%$ of cases, the disease occurs as esophageal squamous cell carcinoma (ESCC) or esophageal adenocarcinoma (EAC). Barrett's adenocarcinoma is the most rapidly increasing cancer in Western countries, while esophageal squamous cell carcinoma (ESCC) dominant in East Asia. Although surgical and medical treatments for ESCC has been improved in recent years, the overall outcome of patients with ESCC is dismal, with a 5year survival rate of less than $10 \%$. And the diagnosis of early stage ESCC is also challenging [1]. Thus, elucidating the molecular mechanisms of ESCC pathogenesis will help to identify specific tumor markers for early detection, risk assessment, and therapeutic target.

MicroRNA (miRNA) is a kind of short non-coding RNAs that suppress the expression of protein coding genes by partial complementary binding, especially to the 3' untranslated regions (UTRs) of messenger RNAs (mRNAs). MiRNA expression alterations are involved in the initiation, progression, and metastasis of human cancer and it is believed that miRNAs function both as tumor suppressors and oncogenes in cancer development $[2,3]$.

C-Myc is a transcription factor acting as a master regulator of genes involved in cell cycle progression, cell growth, differentiation, metabolism and apoptosis. It is also a potent cellular oncogene that is found frequently deregulated in human cancers. This pathological upregulation is frequently due to chromosomal translocations leading to promoter rearrangement [4-7], gene amplification [7] and viral mediated insertional mutagenesis [8]. There is also evidence that c-MYC expression is suppressed by miRNAs $[9,10]$.

In this study, we first detected 6 candidate miRNAs expression in 79 ESCC tissues and found the expression of miR-205, miR-34b and miR-1294 was significantly reduced. By luciferase assay and western bot, we confirmed c-MYC is a direct target of miR-1294. Subsequently, we examined the function of miR-1294 on ESCC cells proliferation, migration and invasion. The correlation between miR-1294 level in tumor and patient prognosis was also analyzed.

\section{Materials and Methods}

Specimen collection

ESCC tissues and matched normal tissues were obtained from patients undergoing primary surgical treatment of esophageal carcinoma from 2009 to 2013 in the Department of Thoracic Surgery of Ningbo Medical Centre, Li Huili Hospital in China. No patient had received preoperative irradiation or chemotherapy. The samples were fresh frozen in liquid nitrogen and stored at $-80^{\circ} \mathrm{C}$ until RNA and protein extraction. The histological diagnosis of tumor was made by a pathologist. Clinical information was also collected and analyzed. Written consent for tissue donation (for research purposes) was obtained from the patients before tissue collection.

\section{Western blotting}

Protein extracts from tissues or cells were boiled in SDS/ $\beta$-mercaptoethanol sample buffer. And $20 \mu \mathrm{g}$ samples were loaded into each lane of $10 \%$ polyacrylamide gels. The proteins were separated by electrophoresis, and the proteins in the gels were then blotted onto PVDF membranes (Amersham Pharmacia Biotech, St. Albans, Herts, UK) by electrophoretic transfer. The membrane was incubated with mouse anti-c-MYC (Abcam, Cambridge, MA, USA)and mouse anti- $\beta$-actin monoclonal antibody (Santa Cruz Biotechnology Inc., Santa Cruz, CA, USA) for $2 \mathrm{~h}$ at $37^{\circ} \mathrm{C}$. The specific protein-antibody complex was detected by horseradish peroxidase conjugated rabbit anti-mouse IgG. Detection of chemiluminescence reaction was carried out using the ECL kit (Pierce, Appleton, WI, USA). The $\beta$-actin signal was used as a loading control.

miRNA RT-qPCR quantification

Quantitive RT-PCR analysis was used to determine the relative expression levels of 5 selected miRNAs. Total RNA was extracted from tissues and cells, using Trizol (Invitrogen, Carlsbad, CA, USA) according to 
Liu et al.: MiR-1294 is Related to ESCC by Targeting C-MYC

the manufacturer's instructions. The expression of candidate miRNAs was detected by TaqMan miRNA RT-Real Time PCR. Single-stranded cDNA was synthesized by TaqMan MicroRNA Reverse Transcription Kit (Applied Biosystems, Foster City, CA, USA) and then amplified by TaqMan Universal PCR Master Mix (Applied Biosystems, Foster City, CA, USA) and miRNA-specific TaqMan MGB probes (Applied Biosystems, Foster City, CA, USA). The U6 snRNA was used for normalization. Each sample was measured in triplicate and the experiment was repeated at least three times for the detection of miRNAs.

\section{Dual Luciferase Reporter Assays}

To generate 3'-UTR luciferase reporter, full length of $389 \mathrm{bp} \mathrm{3'-UTR} \mathrm{from} \mathrm{c-MYC} \mathrm{were} \mathrm{cloned} \mathrm{into}$ the downstream of the firefly luciferase gene in pmirGLO Vector (Promega). MiR-1294 mimic and miR1294 inhibitor were synthesized by GenePharma Co., Ltd (Shanghai, China). pmirGLO vector contain 3'UTR of c-MYC with 4 nucleotides mutation was used to confirmed the binding region of miR-1294. For luciferase reporter assays, HEK293T cells were seeded in 48-well plates. Luciferase reporter vectors were co-transfected with miR-1294 mimic or inhibitor by using lipofectamine 2000 (Invitrogen, Carlsbad, CA USA). Two days later, cells were harvested and assayed with the Dual-Luciferase Assay (Promega, Madison, WI USA). Each treatment was performed in triplicate in three independent experiments. The results were expressed as relative luciferase activity (Firefly LUC/Renilla LUC).

\section{Cell proliferation assay}

EC109 and KYSE-450 cells were seeded in 96-well plates at low density (5x103) in DMEM culture, and allowed to attach overnight. The cells were then transfected with miR-1294 mimics or inhibitor, with scrambled sequence short RNA as control. Twenty microliters MTT (5mg/ml) (Sigma, St. Louis, MO, USA) were added into each well $48 \mathrm{~h}$ after transfection, and the cells were incubated for another $4 \mathrm{~h}$. The absorbance was recorded at A490nm with a 96-well plate reader after the DMSO was added.

\section{Cell migration and invasion assays}

EC109 cells were transfected with the miR-1294 mimic or inhibitor with scramble RNA as control. The cells were transfected for $48 \mathrm{~h}$ and were then harvested and subjected to the subsequent assays. For migration assays, the transfected cells $\left(0.5 \times 10^{6}\right.$ cells $\left./ \mathrm{ml}\right)$ were seeded in the top of an 8.0 -mm-pore membrane chamber (Corning Costar Corp., Cambridge, MA, USA). Following a $12 \mathrm{~h}$ incubation period, cells passed through the membrane to attach to the bottom of membrane were fixed and stained with hematoxylin and eosin (Sigma-Aldrich, St. Louis, MO, USA). Cells were scraped and removed from the top of chamber. Membranes were mounted on cover slides, and cells were counted. Cell migration was quantified by counting the amount of cells passing through the pores from five different fields per sample at 100x selected in a random manner. Cell invasion assays were carried out using modified Boyden chambers in 24 -well tissue culture plates at $1 \times 10^{5}$ cells per well (BD Biosciences, USA). All experiments were performed in duplicate.

\section{Colony Formation Assay using Monolayer Culture}

Colony formation assay in soft agar was carried out based on a modified method [11]. Parental KYSE450 and EC109 cells were transfected by miR-1294 mimic or miR-1294 antagonist for $48 \mathrm{~h}$. Then the cells were separately plated in $60 \mathrm{~mm}$ plates at a density of 1000 cells per well. For each clone, three independent wells were examined. After 2 weeks of incubation at $37^{\circ} \mathrm{C}$ and $5 \% \mathrm{CO}_{2}$, colonies were stained with $0.2 \%$ crystal violet and counted. Meanwhile, the miR-1294 expression in paralleled groups was detected using qRT-PCR.

For monolayer culture, freshly seeded EC109 cells $\left(1 \times 10^{5} /\right.$ well $)$ plated in a 12 -well plate were cultured overnight, and then transfected with miR-1294 mimic or inhibitor. Cells were plated in a 6-well plate $48 \mathrm{~h}$ post-transfection. Colonies were stained with Gentian violate. The experiments were performed in triplicate wells for three times. The results were shown as mean \pm s.e.

\section{Statistical analysis}

The relationship between the expression of miR-1294, the c-MYC protein, and the patient clinical characteristics was analyzed using Student's t-test or a $\chi 2$-analysis. The overall survival curves were plotted according to the Kaplan-Meier method, and the generalized log-rank test was applied to compare the 
Fig. 1. The correlation between expression of miR-1294 and c-MYC protein in ESCC patients. (A) The expression levels of 6 selected miRNAs in clinical tumor and non-tumor samples were detected using stem-loop qRT-PCR. The results were analyzed using Student t-test, and $\mathrm{P}<0.05$ was considered statistically significant. (B) The c-MYC expression in tissue samples were detected using western blot. (C) To evaluate the expression of c-MYC, the band intensity of western blot was semi-quantitatively calculated. The relationship between miR-1294 and MYC in 79 clinical samples of ESCC indicated an inverse correlation (Pearson correlation, $r=-0.299 ; P=0.0079$ ).
A
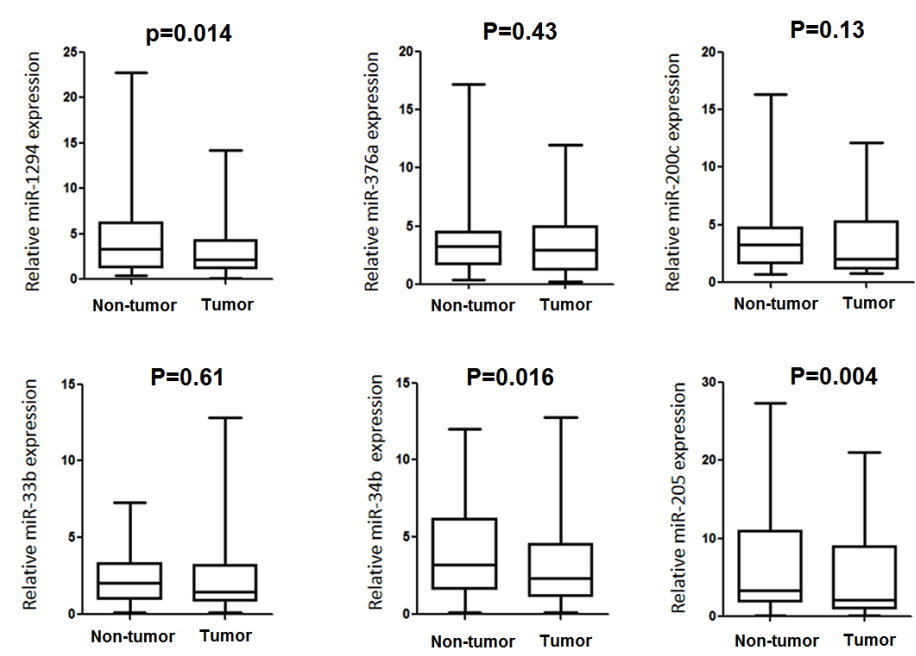

B

C
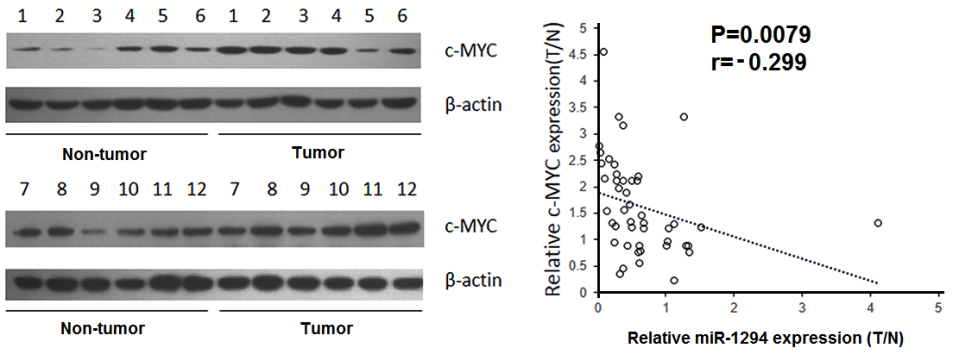

survival curves. The findings were considered to be significant at a $p$-value $<0.05$. All statistical analyses were performed using the SPSS v. 16.0 software program (SPSS, Inc., Chicago, IL, USA).

\section{Results}

Clinical pathological significance of miR-1294 and c-MYC in ESCC patients

C-MYC is a transcription factor that regulates a broad range of cellular processes, which have been recognized to contribute to the initiation and progression of tumors. Overexpressed c-MYC is found in a large part of ESCC patients and is related to poor outcome. MiRNA is a kind of short non-coding RNAs that suppress the expression of protein coding genes by partial complementary binding to the 3'UTR of target gene. Disturbed miRNAs expression also has been found related to ESCC. To unveil the relation between some down regulated miRNAs and the overexpression c-MYC in ESCC, we first analyzed the correlation between the expressions of selected miRNAs and c-MYC. As shown in Fig. 1A, using qRT-PCR, we found the expression of miR-1294, miR-34b and miR-205 was down regulated significantly $(\mathrm{P}=0.014,0.016$ and 0.004$)$. Meanwhile, we examined the c-MYC protein expression level by western blot in the samples from ESCC patients. As shown in Fig1B, the expression of c-MYC is up-regulated in 43 ESCC tissue samples (54.4\%). To evaluate c-MYC expression, the relative band intensity was determined by Quantity One software with $\beta$-actin as loading control. Through analysis on expression of miR-1294, miR-34b and miR-205, we found an inverse correlation between the expression levels of miR-1294 and c-MYC in 79 clinical samples of ESCC. Low levels of miR-1294 were associated with high c-MYC expression (Pearson correlation, r-0.229; P<0.01; Fig. 1C).

We divided the 79 ESCC patients into two groups according to the ratio of their cancer/ normal tissue expression levels of miR-1294, as $\geq 1.0$ or $<1.0$ for the cancer/noncancerous 
Liu et al.: MiR-1294 is Related to ESCC by Targeting C-MYC

Table 1. The miR-1294 expression and clinicopathological characteristics of the patients. ${ }^{*} \mathrm{P}<0.05,{ }^{* *} \mathrm{P}<0.01$

\begin{tabular}{llllll}
\hline Factors & & Total (79) & High (29) & Low (50) & P value \\
\hline Age (mean \pm s.d.) & & & $64.3 \pm 8.5$ & $64.9 \pm 6.7$ & 0.753 \\
Sex & Male & 54 & 23 & 31 & 0.114 \\
& Female & 25 & 6 & 19 & \\
Size & $<40 \mathrm{~mm}$ small & 37 & 19 & 18 & $0.018^{*}$ \\
& $\geq 40 \mathrm{~mm}$ large & 42 & 10 & 32 & \\
Lymph node metastasis & Absent & 25 & 12 & 13 & 0.161 \\
& Present & 37 & 17 & 37 & \\
Lymphatic invasion & Absent & 27 & 14 & 13 & $0.0448^{*}$ \\
& Present & 52 & 15 & 37 & \\
Venous invasion & Absent & 26 & 13 & 13 & $0.0211^{*}$ \\
& Present & 53 & 16 & 37 & \\
Stage & I, II & 44 & 19 & 25 & 0.185 \\
& III, IV & 35 & 10 & 25 & \\
MYC & Positive & 44 & 10 & 34 & $0.0035^{* *}$ \\
& Negative & 35 & 15 & 16 & \\
\hline
\end{tabular}

Fig. 2. Kaplan-Meier curves according to the miR-1294 and c-MYC status. (A) The overall survival curves are presented according to the expression level of miR-1294 in ESCC patients. Patients with low miR-1294 expression had a poorer prognosis than those with high expression. (B) The overall survival curves according to the c-MYC expression level in ESCC patients. The positive c-MYC group had a significantly poorer prognosis for 5-year overall survival than the positive group. ${ }^{*} \mathrm{P}<0.05$, ${ }^{*} \mathrm{P}<0.01$.
A

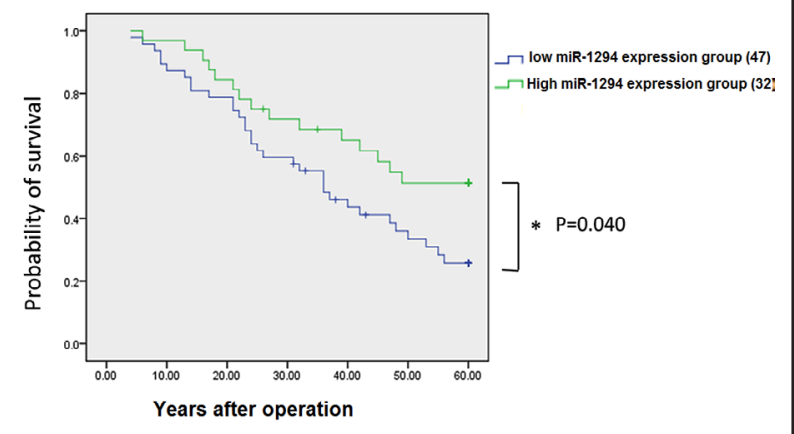

B

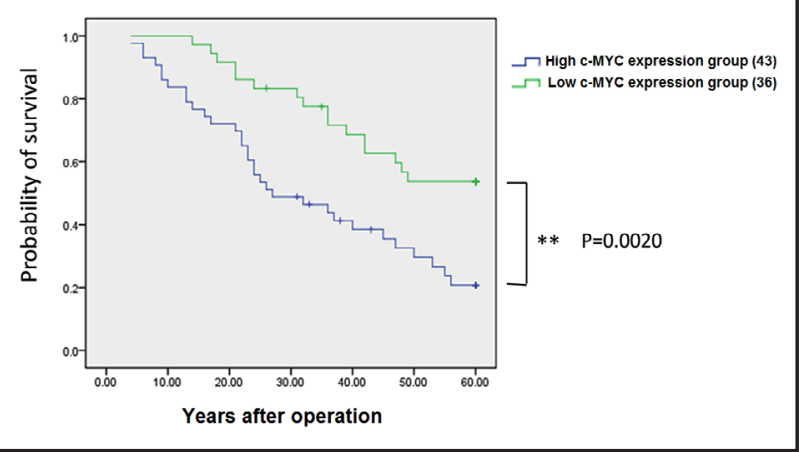

tissues expression levels of miR-1294. There were 29 cases (36.7\%) in the high miR-1294 group and 50 cases (63.3\%) in the low miR-1294 expression group.

The association between the patient clinical characteristics and miR-1294 expression is summarized in Table 1 . There were significant differences in tumor size $(\mathrm{P}=0.018)$, numbers of lymphatic invasion $(\mathrm{P}=0.0448)$ and venous invasion between the groups.

The prognostic significance of miR-1294 and MYC in ESCC

An analysis of 5-year overall survival rate demonstrated that the low miR-1294 expression group had a significantly poorer prognosis than the high expression group 
Fig. 3. The expression of c-MYC is suppressed by miR-1294. (A) Schematic diagram for the predicted interaction between miR-1294 and c-MYC mRNA. Red characters represent the mutant nucleotides. (B) HEK293T cells were co-transfected with miRNA control, miR1294 mimic, anti-miR control or miR-1294 antagonist and pmir-GLO-MYC for dual-luciferase assay (left). When 4 nucleotides of the binding site of miR-1294 in the 3'-UTR of c-MYC were mutated, the luciferase activity was not significantly changed by miR-1294 (right). The results were analyzed by student t-test and $\mathrm{P}<0.05$ was considered as statistically significant. (C) c-MYC protein levels of KYSE-450 and EC109 cells which transfected with miR-1294 mimic or miR-1294 antagonist were detected by western blot. (D) c-MYC mRNA levels were detected by qRT-PCR. The results were analyzed by student t-test and $\mathrm{P}<0.05$ was considered as statistically significant. ${ }^{*} \mathrm{P}<0.05$, $* * \mathrm{P}<0.01$.

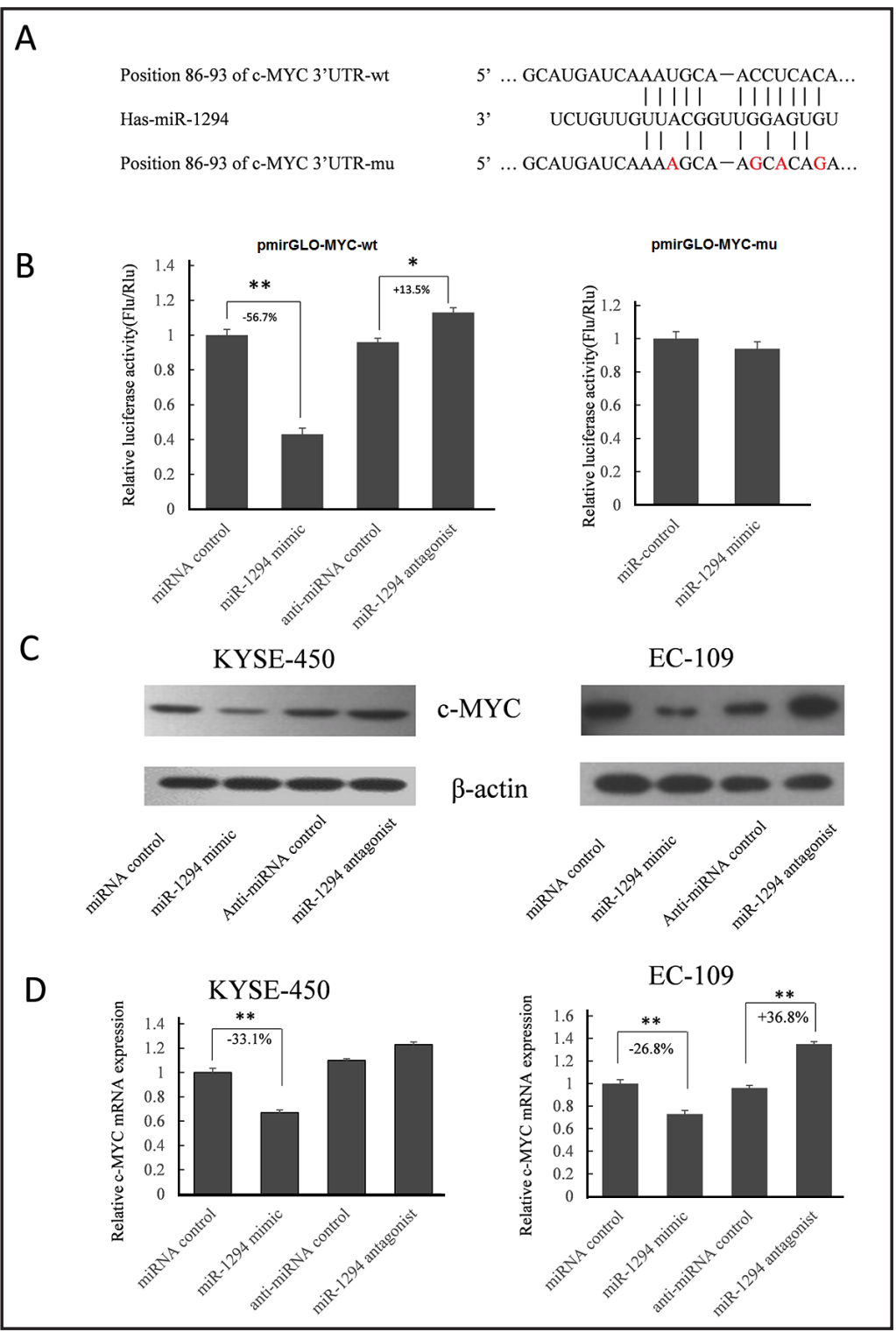

$(\mathrm{P}=0.040$; Fig. 2A). Similarly, the high c-MYC expression group had a significantly poorer prognosis of 5-year overall survival than the low expression group ( $\mathrm{P}=0.0020$; Fig. $2 \mathrm{~B})$.

MiR-1294 suppresses c-MYC expression by direct targeting c-MYC 3'UTR

To investigate mechanisms of the negative correlation between miR-1294 and c-MYC expression, we performed dual-luciferase assay to explore if c-MYC is a direct target gene of miR-1294. Full length of 389bp 3'-UTR from c-MYC were cloned into the downstream of the firefly luciferase gene in pmirGLO Vector to generate pmirGLO-MYC reporter vector. HEK293T cells were co-transfected with pmirGLO-MYC and miR-1294 mimics or antagonist. As shown in Fig. 3B (left), compared with miRNA control, the luciferase activity was significantly suppressed by miR-1294, about $56.7 \%(\mathrm{P}<0.01)$. Furthermore, the luciferase activity was significantly up-regulated by miR-1294 antagonist compared with anti-miR control, about 13.5\% $(\mathrm{P}<0.05)$. These results indicate that miR-1294 targets the 3'-UTR of $\mathrm{c}-\mathrm{MYC}$, leading to the change of firefly luciferase translation.

Seed sequence mutation clone was also used to further confirm the binding site for miR1294 (Fig. 3A). With online bioinformatics tool TargetScan (http://www.targetscan.org) we found a predicted target site existed in the 3'UTR of c-MYC mRNA. We constructed the vector 
A

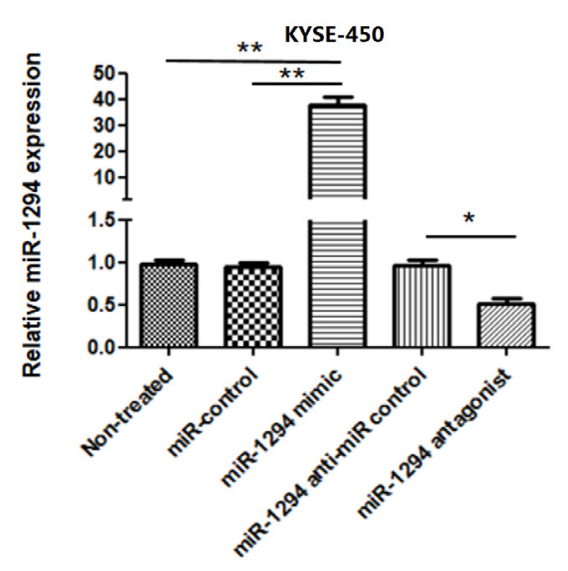

B

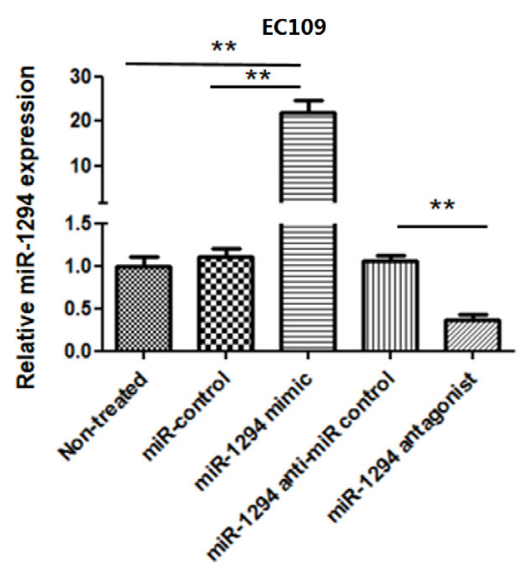

Fig. 4. The expression of miR-1294 in KYSE-450 and EC-109 cells. qRT-PCR was employed to detected the miR-1294 expression in miR-1294 mimic or antagonist treated or non-treated KYSE-450 and EC-109 cells 48 hours post transfection. The results were analyzed using student $\mathrm{t}$-test and $\mathrm{P}<0.05$ was considered statistically significant. $* \mathrm{P}<0.05,{ }^{* *} \mathrm{P}<0.01$.

contains putative miR-1294 binding region in the 3'-UTR of c-MYC with 4 mutant nucleotides (designated as pmirGLO-MYC-mu). The histogram in Fig. 3B (right) showed that the enzyme activity was not significantly reduced in cells transfected with miR-control compared with miR-1294 mimic $(P>0.05)$. These results indicate that miR-1294 may suppress c-MYC expression through binding to seed sequence at the 3'-UTR of c-MYC, and c-MYC may be a direct target gene of miR-1294.

To further examine whether endogenous c-MYC expression is suppressed by miR-1294, EC109 and KYSE-450 cells were transfected with miR-1294 mimics or antagonist. After transfection for 48 hours, the expression of miR-1294 of treated and un-treated EC109 and KYSE- 450 cells were measured by qRT-PCR, and C-MYC protein level was detected by western blot. Compared with control, the miR-1294 level was up-regulated significantly by miR-1294 mimic, about up to 36.4 fold in KYSE-450 cells and 24.3 fold in EC-109 cells; meanwhile, the expression of miR-1294 was down-regulated by miR-1294 antagonist, about $52.1 \%$ in KYSE450 cells and $44.6 \%$ in EC-109 cells (Fig. 4). In the same time, the level of c-MYC protein was significantly suppressed by miR-1294 mimics and up-regulated by miR-1294 antagonist in KYSE-450 (Fig. 3C left) and EC109 cells (Fig. 3C right). The c-MYC mRNA amount was also detected using qRT-PCR. As shown in Fig. 3D, c-MYC mRNA expression was reduced $33.1 \%$ and $26.8 \%$ by overexpressed miR-1294 in KYSE-450 and EC109 cells and went up $36.8 \%$ in EC109 cells. These results indicated that miR-1294 repressed endogenous c-MYC expression in esophageal cancer cells by directly cutting c-MYC mRNA.

MiR-1294 suppresses esophageal cancer cells proliferation, migration and invasion

To further test whether miR-1294 may execute tumor-suppressive functions by targeting c-MYC, we investigated the effect of miR-1294 mediated cell proliferation by MTT assay in EC109 (Fig. 5A) and KYSE-450 cells (Fig. 5B). The cell proliferation ability was significantly reduced by miR-1294 mimic, about 33.8\% in KYSE-450 cells and 48.1\% in EC109 cells. Meanwhile, the cell proliferation was significantly up-regulated by miR-1294 antagonist in KYSE-450 cells, about $22.1 \%$. To further confirm whether miR-1294 repress cell proliferation by targeting MYC, we co-transfected c-MYC expression vector with miR-1294 mimic into KYSE-450 and EC109 cells. As shown in Fig. 5A, B (right), c-MYC expression vector can partially rescue the repressed cell proliferation caused by miR-1294 overexpression, which means miR-1294 suppress cells proliferation partially through targeting c-MYC. 
Fig. 5. Downregulated miR1294 is related to enhance esophageal cancer cells proliferation and colony formation. (A, B) EC109 and KYSE-450 cells were seeded in 96-well plates at low density $\left(5 \times 10^{3}\right)$ in DMEM culture, and allowed to attach overnight. The cells were then transfected with miR1294 mimics or inhibitor, with scrambled sequence short RNA as control. MTT assay was performed 48 hours post transfection. The results were analyzed using student t-test and $\mathrm{P}<0.05$ was considered statistically significant. (C, D) KYSE-450 and EC109 cells were transfected by miR-1294 mimic or miR-1294 antagonist. $48 \mathrm{~h}$ later, the cells were separately plated in $60 \mathrm{~mm}$ plates at a density of 1000 cells per well. For each clone, three independent wells were examined. After 2 weeks of incubation at $37^{\circ} \mathrm{C}$ and 5\% $\mathrm{CO} 2$, colonies were stained with $0.2 \%$ crystal violet and counted. The results were analyzed using student t-test and $\mathrm{P}<0.05$ was considered statistically significant. ${ }^{*} \mathrm{P}<0.05$, $* * \mathrm{P}<0.01$.

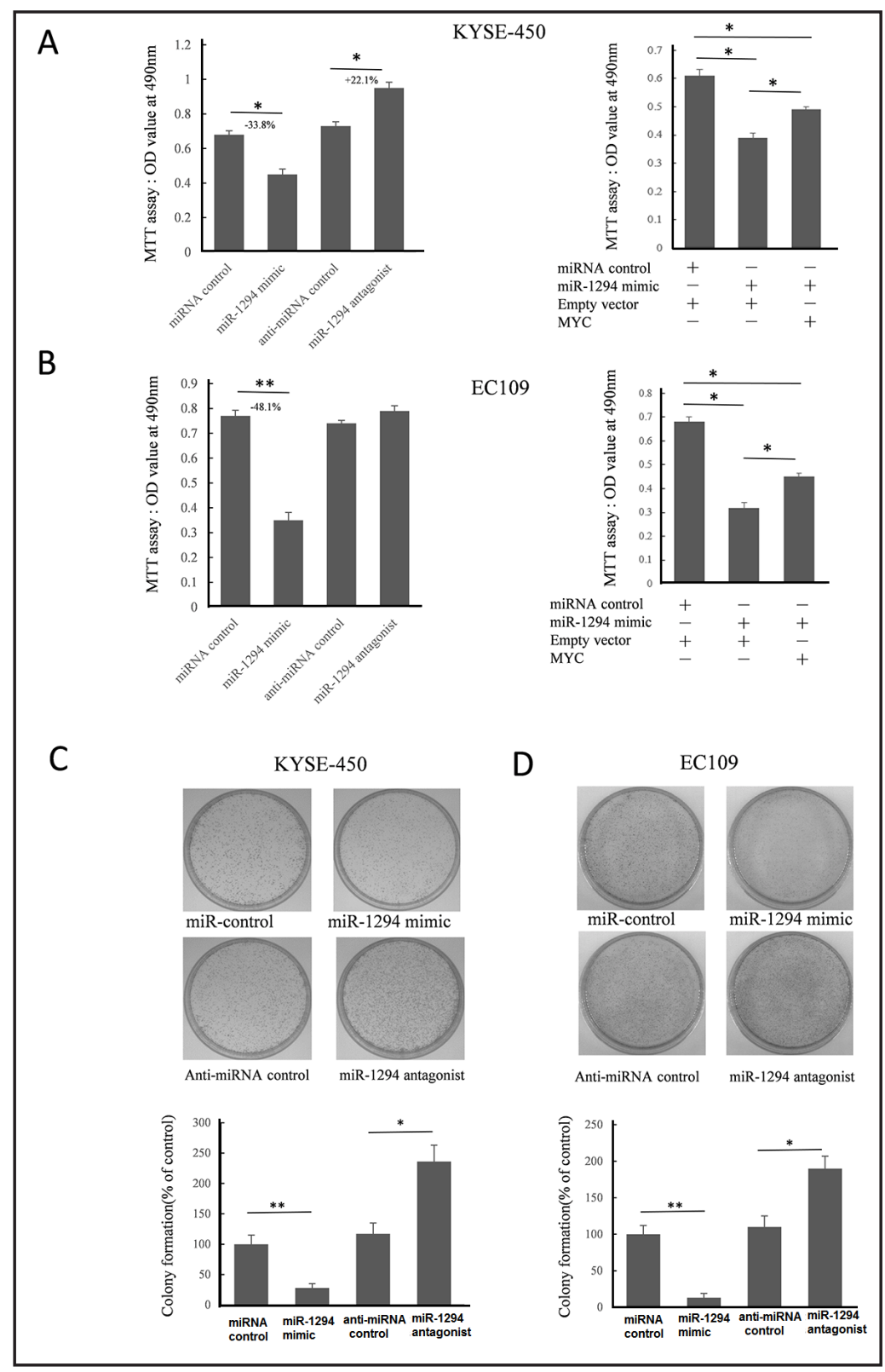

The antitumor function of miR-1294 was also examined using colony formation assay. Cells were transfected with miR-1294 mimic or antagonist with scramble sequenced double or single strands short RNA as control. After 2 week's culture, miR-1294 mimic groups formed colonies smaller in size and fewer in number than miR-control. Conversely, miR1294 antagonist groups formed colonies larger in size and more in number than anti-miRNAcontrol (Fig. 5C, D).

In order to further investigate the role of miR-1294 in controlling the metastasis of esophageal cancer cells, we analyzed the effects of miR-1294 on the migratory and invasive behavior of esophageal cancer cells. As shown in Fig. 6, the migration and invasion capacity was significantly suppressed in KYSE-450 cells transfected with miR-1294 compared with miR-control $(\mathrm{P}<0.05)$. In contrary, the KYSE-450 cells migration and invasion ability were significantly enhanced by miR-1294 antagonist. These findings suggest that the level of miR1294 is closely associated with metastasis of esophageal cancer cells.

MYC is a transcription factor that regulates the expression of a large group of genes, including many microRNAs $[12,13]$. To investigate whether the expression of miR-1294 
Fig. 6. Downregulated miR-1294 is related to enhanced esophageal cancer cells migration and invasion. EC109 cells were transfected with the miR-1294 mimic or inhibitor with scramble RNA as control for $48 \mathrm{~h}$. The transfected cells were harvested and subjected to the following assays. (A) For migration assays, the transfected cells $(0.5 \times 106$ cells $/ \mathrm{ml})$ were seeded in the top of an $8.0 \mathrm{~mm}$-pore membrane chamber. Following a 12 $\mathrm{h}$ incubation period, cells that passed through the membrane to attach to the bottom of membrane were fixed and stained with hematoxylin and eosin. Cells were scraped and removed from the top of chamber. Membranes were mounted on cover slides, and cells were counted. Cell migration was quantified by counting the amount of cells passing through the pores from five different fields per sample at $100 \times$ selected in a random manner. (B)

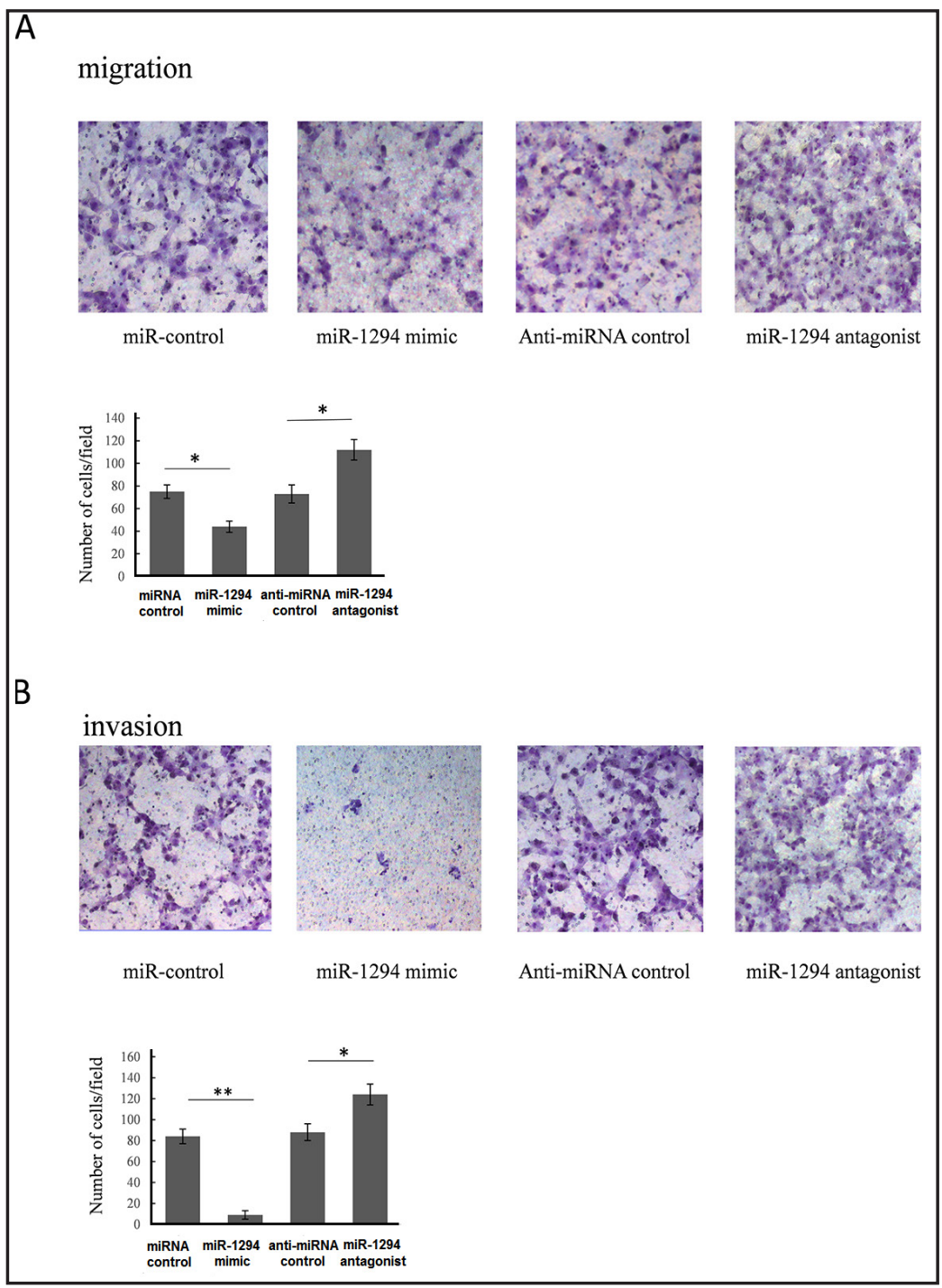

Cell invasion assays were carried out using modified Boyden chambers in 24-well tissue culture plates at $1 \times 10^{5}$ cells per well. All experiments were performed in duplicate. The results were analyzed using student t-test and $\mathrm{P}<0.05$ was considered statistically significant. ${ }^{*} \mathrm{P}<0.05,{ }^{* *} \mathrm{P}<0.01$.

is regulated by MYC, we constructed the MYC expression vector. As shown in Fig. 7, the expression of miR-1294 was up-regulated significantly. However, there is no significant change on the miR-1294 expression. Subsequently, we detected the expression of miR-17 which is a direct target of MYC, and analyzed the correlation between miR-17 and miR-1294. As shown in Fig. 8, a negative correlation existed in the expression of miR-17 and miR-1294.

\section{Discussion}

In the present study, it was found that miR-1294 was significantly down-regulated in human ESCC tissue compared with the corresponding normal tissue $(\mathrm{P}=0.014)$. And patients with low miR-1294 expression had a significantly poorer prognosis than those with high miR-1294 expression $(\mathrm{P}=0.040)$. We also provided evidence that a negative association exists between the expression of miR-1294 and c-MYC expression in ESCC patients (Pearson correlation, $\mathrm{r}=-0.299, \mathrm{P}=0.0079)$. Additionally, we confirmed that miR-1294 suppress esophageal cancer cells proliferation, migration and invasion capacity through targeting 
Fig. 7. MYC does not regulate miR-1294 expression. Cells were transfected with MYC expression or empty control vector. 48 hours after transfection, the expression of miR-1294 was detected by RT-PCR, and MYC was detected by western blot. The results were analyzed using student t-test and $\mathrm{P}<0.05$ was considered statistically significant.
A
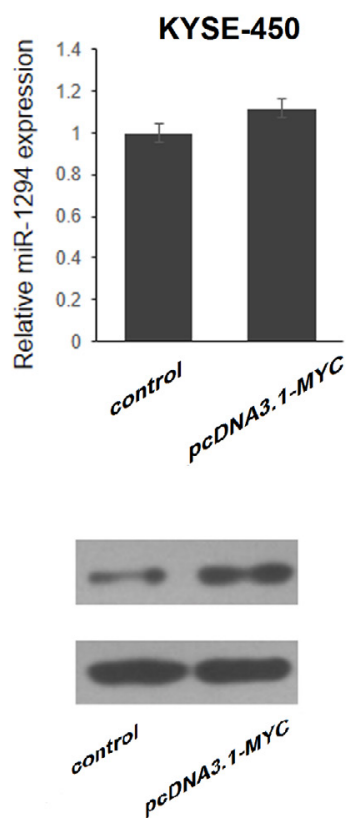

B

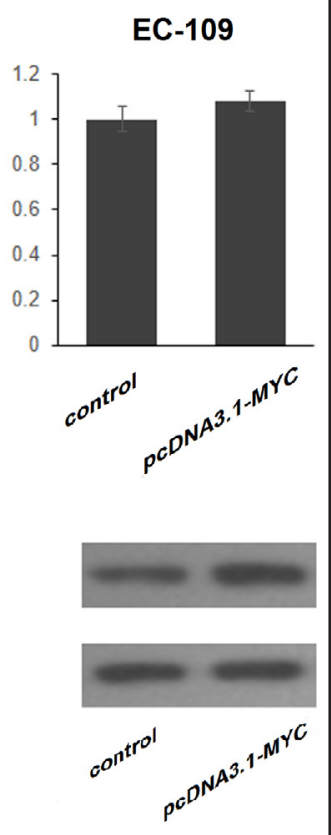

Fig. 8. Correlation between the expression of miR1294 and miR-17. The expression of miR-1294 and miR-17 was detected by qRT-PCR. The relationship between miR-1294 and miR-17 expression in 79 clinical samples of ESCC indicated an inverse correlation (Pearson correlation, $r=-0.385 ; \mathrm{P}=0.0091$ ).

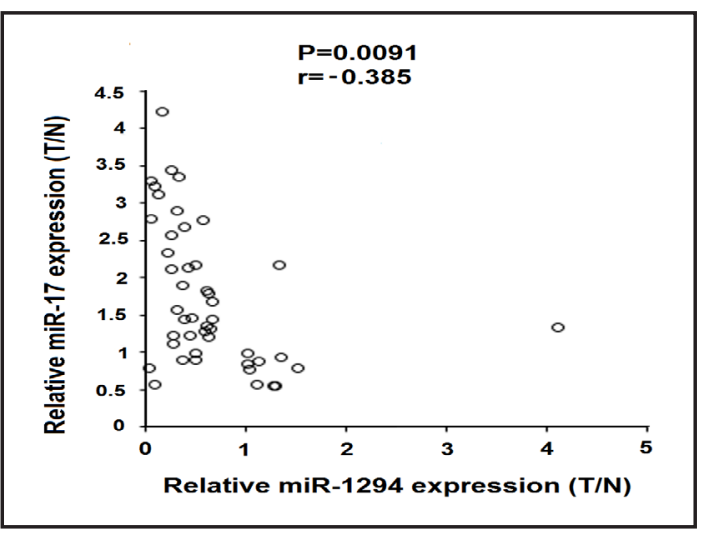

c-MYC in vitro. These findings suggested that the down-regulation of miR-1294 correlates with poor prognosis of ESCC, which is possibly due to reduced repression on the function of c-MYC protein.

C-MYC is one of the most prevalent oncogenes altered in human cancer. It is deregulated in about $50 \%$ of tumors, especially in Burkitt lymphoma, and also existed in cervix, colon, breast, lung cancer. Although there are some studies reported that the positive expression of c-MYC was significantly correlated with poor prognosis, the underlying mechanisms were not well understood $[14,15]$. As disturbed miRNAs expression is also related to ESCC pathogenesis, our study started with detecting the expression of six selected miRNAs which may suppress c-MYC expression. We found three miRNA: miR-1294, miR-230 and miR205 had reduced expression in ESCC tissue samples. And only the expression of miR-1294 had a negative correlation with c-MYC expression, which suggests c-MYC may be a direct target gene of miR-1294. Detected by dual-luciferase assay, western blot and qRT-PCR, we confirmed that miR-1294 repressed c-MYC expression by directly targeting 3'UTR of c-MYC mRNA though a speed up mRNA degradation way.

Till now, little was known about the function of miR-1294. Since c-MYC function is closely related to cancer cells proliferation, migration and invasion, we examined the biological function of miR-1294 in these aspects. We found that miR-1294 function as a 
tumor suppressor through repressing esophageal cancer cells proliferation, migration and invasion in vitro.

Although we suggest that miR-1294 is an anti-tumor miRNA by targeting c-MYC in ESCC patients, the full function of miR-1294 is still need to be further investigated because of the complex mode of action of miRNA. It is considered that one miRNA may repress hundreds to thousands of genes directly, and one gene may be suppressed by tens to hundreds of miRNAs. Meanwhile, the expression and function of miRNAs has tissue specificity, so the roles of miR-1294 needed to be further unveiled.

In conclusion, the present study indicates that low expression level of miR-1294 has a significant correlation with poor prognosis of ESCC patients through promotion of the function of c-MYC.

\section{Disclosure Statement}

None.

\section{References}

1 Holmes RS, Vaughan TL: Epidemiology and pathogenesis of esophageal cancer. Semin Radiat Oncol 2007;17:2-9.

-2 2 Wu WK, Lee CW, Cho CH, Fan D, Wu K, Yu J, Sung JJ: Microrna dysregulation in gastric cancer: A new player enters the game. Oncogene 2010;29:5761-5771.

-3 Nicoloso MS, Spizzo R, Shimizu M, Rossi S, Calin GA: Micrornas--the micro steering wheel of tumour metastases. Nat Rev Cancer 2009;9:293-302.

-4 Shen-Ong GL, Keath EJ, Piccoli SP, Cole MD: Novel myc oncogene rna from abortive immunoglobulin-gene recombination in mouse plasmacytomas. Cell 1982;31:443-452.

5 Dalla-Favera R, Bregni M, Erikson J, Patterson D, Gallo RC, Croce CM: Human c-myc onc gene is located on the region of chromosome 8 that is translocated in burkitt lymphoma cells. Proc Natl Acad Sci U S A 1982;79:7824-7827.

6 Taub R, Kirsch I, Morton C, Lenoir G, Swan D, Tronick S, Aaronson S, Leder P: Translocation of the c-myc gene into the immunoglobulin heavy chain locus in human burkitt lymphoma and murine plasmacytoma cells. Proc Natl Acad Sci U S A 1982;79:7837-7841.

7 Dalla-Favera R, Wong-Staal F, Gallo RC: Onc gene amplification in promyelocytic leukaemia cell line hl-60 and primary leukaemic cells of the same patient. Nature 1982;299:61-63.

-8 Hayward WS, Neel BG, Astrin SM: Activation of a cellular onc gene by promoter insertion in alv-induced lymphoid leukosis. Nature 1981;290:475-480.

-9 Takwi AA, Li Y, Becker Buscaglia LE, Zhang J, Choudhury S, Park AK, Liu M, Young KH, Park WY, Martin RC, Li Y: A statin-regulated microrna represses human c-myc expression and function. EMBO Mol Med 2012;4:896-909.

-10 Choi YJ, Lin CP, Ho JJ, He X, Okada N, Bu P, Zhong Y, Kim SY, Bennett MJ, Chen C, Ozturk A, Hicks GG, Hannon GJ, He L: Mir-34 mirnas provide a barrier for somatic cell reprogramming. Nat Cell Biol 2011;13:13531360.

11 Li Y, Tondravi M, Liu J, Smith E, Haudenschild CC, Kaczmarek M, Zhan X: Cortactin potentiates bone metastasis of breast cancer cells. Cancer Res 2001;61:6906-6911.

12 Li Y, Choi PS, Casey SC, Dill DL, Felsher DW: Myc through mir-17-92 suppresses specific target genes to maintain survival, autonomous proliferation, and a neoplastic state. Cancer cell 2014;26:262-272.

-13 Jackstadt R, Hermeking H: Micrornas as regulators and mediators of c-myc function. Biochim Biophys Acta 2014;pii: S1874-9399(14)00080-7.

14 Miyawaki Y, Kawachi H, Ooi A, Eishi Y, Kawano T, Inazawa J, Imoto I: Genomic copy-number alterations of myc and fhit genes are associated with survival in esophageal squamous-cell carcinoma. Cancer Sci 2012;103:1558-1566.

15 Wang W, Xue L, Wang P: Prognostic value of beta-catenin, c-myc, and cyclin d1 expressions in patients with esophageal squamous cell carcinoma. Med Oncol 2011;28:163-169. 
This is an Open Access article licensed under the terms of the Creative Commons AttributionNonCommercial 3.0 Unported license (CC BY-NC) (www.karger.com/OA-license), applicable to the online version of the article only. Distribution permitted for non-commercial purposes only.

\section{Erratum}

In the article by Liu et al., entitled "Down-Regulation of MiR-1294 is Related to Dismal Prognosis of Patients with Esophageal Squamous Cell Carcinoma through Elevating C-MYC Expression” [Cell Physiol Biochem 2015;36:100-110 (DOI: 10.1159/000374056], is a printing error in the affiliations and correspondig author address.

The corrected affiliations and the corresponding author address are stated correctly here:

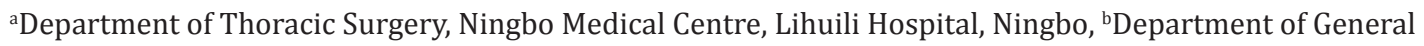
Surgery, The Second Affiliated Hospital\& Yuying Children's Hospital of Wenzhou Medical University, Wenzhou, 'Department of Thoracic Surgery First Affiliated Hospital of Zhejiang University, Hangzhou, China

Xiayi Lv

Department of Thoracic Surgery, First Affiliated Hospital of Zhejiang

University. No 79. Qingchun Road, Hangzhou 310003 (China)

E-Mail Ivxiay@163.com 